\title{
Análisis de la Modulación Arquitectónica en Edificaciones Menores de CLT; estudio de casos.
}

\section{Analysis of Architectural Modulation in Minor Buildings of CLT; cases study}

Rodrigo García-Alvarado ${ }^{(*)}$, Ignacio Rojas-Wilckens ${ }^{(* *)}$, Mauricio Vargas-Mosqueda ${ }^{(* *)}$

\section{RESUMEN}

La construcción con tableros contralaminados de madera (CLT) posee relevantes capacidades de prefabricación y desempeño ambiental, por lo que se ha comenzado a aplicar en algunas edificaciones. Este trabajo analiza las condiciones de modulación arquitectónica de construcciones de pequeño tamaño en CLT, con el fin de determinar estrategias de diseño y de estandarización productiva que fomenten su utilización. Se revisan primero características de los tableros, y luego dimensiones arquitectónicas en una muestra internacional de casos, identificando convergencias en las magnitudes y ordenaciones, así como una versatilidad de diseño y expresión espacial. Estas propiedades revelan la posibilidad de focalizar la producción de tableros en las dimensiones y configuraciones más utilizadas, para facilitar su elaboración y aplicación, como también diversificar disposiciones arquitectónicas para promover esta tecnología constructiva.

Palabras clave: Construcción Industrializada; Arquitectura en Madera; Tableros Contralaminados de Madera; Diseño Modular.

\section{ABSTRACT}

Construction with counter-laminated timber (CLT) boards has substantial capacities in prefabrication and environmental performance, as it has begun to apply to some buildings. This work analyzes the conditions of architectural modulation in small CLT-constructions, in order to determine the design strategies and production standardization that an impell its use. First, productive characteristics, and then architectural dimensions of an international sample of cases are reviewed, identifying convergences in the magnitudes and ordinances, as well as a significant versatility of design and spatial expression. These properties reveal the possibility of focusing on the production in the dimensions and configurations more used, to facilitate its elaboration and implementation, as well as to diversify architectural arrangements to disseminate this constructive technology

Keywords: Industrialized Construction; Timber Architecture; Counter-laminated Timber Boards; Modular Design.

$\left(^{*}\right)$ Doctor Arqto. Profesor Titular, Depto. de Diseño y Teoría de la Arquitectura, Universidad del Bío-Bío, Concepción (Chile)

$\left.{ }^{* *}\right)$ Arquitecto, Magister en Construcción en Madera, Docente, Depto. de Diseño y Teoría de la Arquitectura y Depto. de Ciencias de la Construcción, Universidad del Bío-Bío, Concepción (Chile).

$\left.{ }^{* *}\right)$ Arquitecto, Magister en Construcción en Madera, Profesor Asistente, Depto. de Ciencias de la Construcción, Universidad del Bío-Bío, Concepción (Chile).

Persona de contacto/Corresponding author: rgarcia@ubiobio.cl (R. García-Alvarado)

ORCID: http://orcid.org/oooo-0oo3-2216-2388 (R. García-Alvarado); https://orcid.org/oooo-ooo2-6532-3455(I. Rojas-Wilckens); http://orcid.org/ oooo-0oo2-4936-4249 (M. Vargas-Mosqueda).

Cómo citar este artículo/Citation: Rodrigo García-Alvarado, Ignacio Rojas-Wilckens, Mauricio Vargas-Mosqueda (2021). Análisis de la Modulación Arquitectónica en Edificaciones Menores de CLT; estudio de casos. Informes de la Construcción, 73(562): e387. https://doi.org/10.3989/ ic. 77708

Copyright: (C) 2021 CSIC. Este es un artículo de acceso abierto distribuido bajo los términos de la licencia de uso y distribución Creative Commons Reconocimiento 4.0 Internacional (CC BY 4.0). 


\section{INTRODUCCIÓN}

El desarrollo de tableros de madera contralaminada (CLT) ha hecho emerger una nueva tecnología constructiva, que tiene diversas ventajas productivas y ambientales $(1,2)$. Los tableros CLT son paneles formados por piezas longitudinales de madera aserrada o madera laminada encolada, adheridas por los costados y dispuestas en capas perpendiculares entre sí, constituyendo un bloque superficial macizo (Fig.1). Los tableros CLT se pueden utilizar en edificaciones como muros soportantes, divisorios o como revestimientos; así como suelos, entrepisos o techumbres. Las placas se colocan ensambladas entre si o mediante conectores metálicos, como también combinadas con otras piezas estructurales (vigas o pilares metálicos, de madera entera o laminada), además de considerar distintas coberturas e instalaciones. Los tableros CLT pueden proveer resistencia estructural, masa térmica, aislación acústica, térmica e ignifuga $(3,4,5)$, con un adecuado desempeño en el ciclo de vida (6), por lo que se ha aplicado especialmente en varias edificaciones mayores y de mediana altura $(1,2)$. Además, permiten la prefabricación de componentes, facilitando el transporte, productividad y adaptación (7). Sin embargo, en muchos países aún es una tecnología incipiente, con escasa producción y sistematización industrial, que requiere ampliar sus proyecciones comerciales y procesos constructivos (2), especialmente para edificaciones menores, que poseen una amplia diversidad, pero esfuerzos limitados de producción y un escaso estudio.

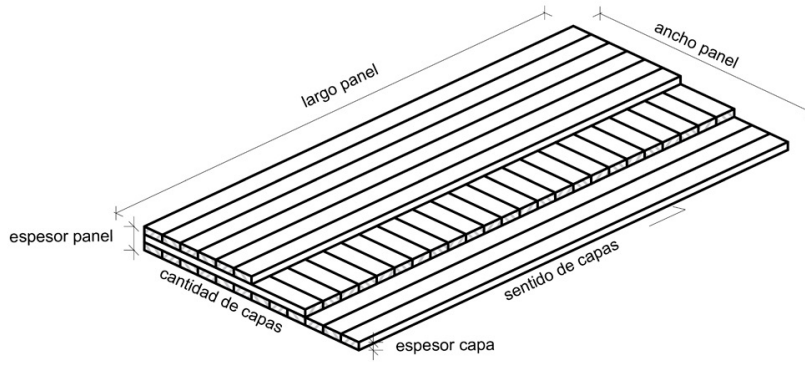

Fig.1. Conformación de tablero CLT (fuente: elaboración propia).

Por esta razón, el presente trabajo revisa condiciones de diseño arquitectónico de los edificios de pequeñas dimensiones con tableros CLT, para facilitar la elaboración de construcciones con esta nueva tecnología, analizando el dimensionamiento y ordenación geométrica de edificios realizados en los últimos 8 años en distintas partes del mundo, para esclarecer las estrategias formales que poseen estas construcciones. Esto se hace mediante una selección y análisis morfológico de construcciones de tamaños reducido (menos de $400 \mathrm{~m} 2$ construidos), ejecutadas mayormente con tableros CLT, para determinar sus condiciones de aplicación.

\section{Modulación Arquitectónica}

El proyecto arquitectónico reconoce desde la época clásica la utilización de reglas geométricas para definir disposiciones constructivas y funcionales de los edificios (8). Primeramente, se identificaron repeticiones o proporciones entre las medidas exteriores de los volúmenes, en los interiores de recintos o partes de los elementos constructivos. Como también relativas al distanciamiento de los componentes, ya sea en horizontal (en planta) o vertical (en alzados o secciones). En el Renacimiento, y en especial durante la Ilustración, se reconocieron tramas y modulaciones de los edificios, consideradas como la reiteración de las líneas centrales de los elementos, en que se advierten sus dimensiones intermedias y el total de distancias (9). De este modo se componen grillas reguladoras, que identifican módulos interiores formados por las distancias transversales. Estas magnitudes se interpretan como elementos geométricos similares (módulos rectangulares, y ocasionalmente triangulares, hexagonales o circulares), que permiten definir series y composiciones de figuras repetidas o variables, como una estrategia de racionalización del diseño y de la planeación constructiva, para facilitar la ejecución e instalación de los elementos constructivos $(10,11)$.

La revolución industrial impulsó luego una producción masiva, que repercutió en la edificación a través de la creciente utilización de elementos prefabricados (vigas, pilares, tabiques, puertas, ventanas, etc.). Lo que incentivó una mayor precisión y coordinación dimensional de la construcción, tanto en el diseño $(12,13)$, como en la industria de productos y la ejecución en terreno. También se impulsó la definición de tolerancias dimensionales, modulaciones variables o jerarquizadas, o incluso de trazados diagonales o de tramos curvos. A pesar que todavía se mantienen algunos elementos o equipos con dimensiones bajo el sistema métrico, y otros con el sistema imperial (pies y pulgadas), lo que dificulta compatibilizarlos. Además, que la diversidad de condiciones, faenas y productos utilizados en la construcción ha limitado una mayor racionalización $(10,14)$. Considerando en la mayoría de las construcciones una cantidad parcial de elementos prefabricados; o en otras, con una ocupación mayor de productos industrializados se aplican diseños regulares y repetitivos. El reciente surgimiento de sistemas digitales de diseño y gestión de las obras, así como maquinarias e instrumentales de mayor precisión han incrementado las capacidades de dimensionamiento y coordinación (15), pero aún se carece de estrategias formales propias de los sistemas constructivos. Los distanciamientos de elementos se originan principalmente por las capacidades resistentes de los componentes y los requerimientos funcionales de los recintos, así como por el dimensionamiento productivo de los elementos y sus variaciones menores.

\section{Modulación de Sistemas Constructivos}

La aplicación de un sistema prefabricado en un proyecto arquitectónico, o de algunos elementos parciales, requiere adecuar algunas dimensiones relevantes del diseño a las medidas de los componentes y sus ordenaciones, estableciendo una coordinación modular entre las magnitudes de los elementos prefabricados y sus distanciamientos soportantes admisibles (16). Esta relación se organiza usualmente a través de tramas horizontales o verticales, cuando constituye elementos portantes, o simplemente a través de consideraciones particulares de medidas para insertar componentes (17). En los programas de diseño digital se pueden establecer grillas de trabajo para facilitar esta disposición, como también elementos determinados (bloques CAD o familias BIM) con medidas fijas o variables de acuerdo a condiciones propias (18). Sin embargo, los distanciamientos e incluso medidas específicas definidas por los productores son diversas y escasamente reconocidas en el trabajo de los proyectistas. Usualmente se aplican ordenaciones particulares en cada proyecto, dificultando la producción estandarizada, aunque suelen repetirse algunas condiciones según el sistema constructivo o el tipo de ocupación del edificio. También se consideran a veces distintos márgenes de dimensiones o 
variaciones a pedido que amplían las posibilidades, pero debilitan la regulación del diseño y complejizan su ejecución. Tampoco se dispone en los sistemas digitales de dibujo o gestión, de tramas tridimensionales, con variaciones especificas o relacionadas con componentes; pero pueden ser programadas mediante lenguajes internos (como Dynamo o Python en Revit).

La industrialización de la construcción requiere una racionalización de los procesos y eficiencia entre las fases de diseño, ingeniería, manufactura y montaje para cumplir los requerimientos específicos de cada proyecto en tiempos rentables. Asimismo, se debe garantizar la autonomía de diseño y flexibilidad arquitectónica y de construcción (19). Uno de los enfoques para esta problemática es la Personalización Masiva (PM), concepto que asume la manufactura industrializada con una variación de conjuntos o aspectos específicos para su adecuación individual (20). Otro concepto considerado es la modularidad del producto (MP) que permite la configuración de múltiples elementos o disposiciones a pedido a partir de un número definido de piezas o componentes prefabricados. Bajo este enfoque, un producto es decompuesto en bloques (módulos) con interfaces específicas que soportan cada configuración. Sin embargo, aún es limitada su aplicación en la arquitectura, de manera que aún no existe medidas modulares ni un método sistemático ampliamente adoptado. De hecho, se afirma que el enfoque de MP utilizado en los procesos de manufactura no puede aplicarse de igual forma en la construcción industrializada ya que constituyen problemas de naturalezas distintas, cuyas diferencias radican principalmente en la doble función del producto arquitectónico, tanto de soporte físico-material del espacio, como de generador del vacío espacial en sí mismo, con condiciones de resistencia mecánica y propiedades físicas que garanticen la habitabilidad (Da Rocha, 2015) (20). En el caso específico de viviendas, si bien los proyectos de construcción se rigen por complejos conjuntos de requisitos que resultan en productos únicos, hay denominadores comunes en estos requisitos a partir de los cuales el producto puede desglosarse en subsistemas y componentes, generando así plataformas de productos modularizadas. Los métodos de diseño para la Personalización Masiva basadas en objetos arquitectónicos pueden apoyar el desarrollo de sistemas de construcción más flexibles (21), como ordenciones de espacios constructivos y actividades de usuarios definidas funcional y experimentalmente, los cuales permitirían un manejo más eficiente del proyecto de diseño y construcción, siendo que los módulos pueden tener diferentes propiedades en diferentes fases del proceso del edificio. Un módulo puede ser integral desde el punto de vista arquitectónico, pero modular en el punto de vista de la ingeniería y producción. Por otro lado, es importante destacar que para garantizar la modularización en la construcción resulta importante la definición de interfaces que permitan la intercambiabilidad de módulos o componentes en función de garantizar múltiples combinaciones (22). El grado en que la interface entre módulos sigue un estándar, garantiza, tanto su compatibilidad e interconexión, como un mayor número de combinaciones. La definición de interfaces entre módulos depende del tipo de interacción entre ellos y estas pueden definirse en categorías como: espaciales, de intercambio energético, de intercambio de información, de intercambio de material y de geometría, donde se establecen criterios de montaje, de forma, talla y tolerancia. En conclusión, la modularidad, desde sus distintos enfoques, resulta una temática importante para garantizar racionalización de la construcción y una cierta autonomía de diseño.
Por esta razón, el presente artículo pretende recoger las reglas dimensionales de la tecnología CLT, a partir de sus condiciones materiales, como también por medio de ejemplos de aplicación que expresan la utilización del sistema por diferentes proyectistas y constructores que integran los procedimientos y requerimientos reales de la edificación, con el fin de sugerir ordenaciones geométricas que se pueden establecer en sistemas de diseño digital para orientar la proyectación arquitectónica con esta tecnología, y así promover su utilización, alcanzado las ventajas productivas y sustentables de este sistema constructivo. Considerando que los usos iniciales de placas CLT en edificios se ha abocado mayormente a grandes edificaciones $(1,2)$, este trabajo se concentra en edificaciones menores que han surgido en los últimos años, debido que otorgan un campo de aplicación amplio y diverso, con esfuerzos limitados de estandarización, carecen de estudios específicos y presentan una particular expresión arquitectónica.

\section{MATERIALES Y METODOS}

Este trabajo, orientado analizar la modulación arquitectónica de construcciones pequeñas en CLT, se desarrolla con una metodología exploratoria en base a documentación disponible, con el fin de realizar una prospección y fomentar la utilización de este sistema constructivo, de manera complementaria a estudios experimentales de aplicación. Esto se lleva a cabo a partir de la revisión de construcciones ejecutadas que expresan una motivación y capacidad existente, a partir de su difusión pública y representación gráfica, que también revelan un reconocimiento e interpretación técnica. El análisis se realiza primero mediante una síntesis de condiciones de aplicación constructiva de los tableros CLT, basado en documentos difundidos que detallan características técnicas. Luego, se recopilan ejemplos arquitectónicos utilizando medios generales de información digital, y se recogen antecedentes, imágenes y planos descriptivos. Posteriormente se efectúan registros y mediciones comparativas de los documentos, reuniendo los datos y valores en tablas que resumen y cuantifican la información. Finalizando con el análisis y discusión general respecto a las intenciones del trabajo.

Los edificios estudiados fueron seleccionados de manera complementaria a una prospección inicial en ámbitos profesionales y organizaciones locales, a través de una búsqueda por internet en Septiembre 2019, utilizando palabras clave en español e inglés (como CLT, arquitectura, edificio, etc.) para recopilar sitios web que presentarán información de construcciones individuales que expresarán poseer superficies inferiores a $400 \mathrm{~m} 2 \mathrm{y}$ haber sido ejecutadas en tableros CLT como elementos portantes (al menos de un nivel ocupable, en algunos casos con niveles inferiores de otro material). La información recolectada debía incluir al menos datos básicos de ubicación, destino, ejecución y responsabilidad proyectual; fotografías desde distintos puntos de vista y planos arquitectónicos completos (plantas, secciones, alzados). En algunos casos se reunieron antecedentes de la misma obra a partir de distintos sitios de internet y se efectuaron consultas a las oficinas proyectistas solicitando información. En varios proyectos se obtuvieron detalles y planos de ejecución de los tableros; en otros casos se interpretó esta información de fotografías de ejecución o respuesta de los proyectistas o ejecutores. El registro de información permitió seleccionar 18 edificios realizados en los últimos 8 años de distintos países (Fig.2), lo que se consideró una muestra adecuadamente representativa de la aplicación de esta tecnología, para analizar con las capacidades disponibles (Tabla 1). 
Tabla 1. Lista de Edificios Analizados.

\begin{tabular}{|c|c|c|c|c|c|c|c|}
\hline $\mathbf{N}^{\circ}$ & Nombre & Ciudad & Pais & Año & \begin{tabular}{|c|} 
Superficie \\
$\left(\mathbf{m}^{2}\right)$
\end{tabular} & Arquitectos & Destino \\
\hline 1 & Dyson Institute & Wiltshire & Reino Unido & 2019 & 32,00 & Wilkinson Eyre & Vivienda \\
\hline 2 & Minimod, Catucaba & Catucaba & Brasil & 2015 & 42,00 & MAPA & Vivienda \\
\hline 3 & Minimod, Catucaba 2 & Catucaba & Brasil & 2015 & 42,00 & MAPA & Vivienda \\
\hline 4 & Casa 2020 & Ortigosa & España & 2014 & 60,00 & Aritz Gonzalez & Vivienda \\
\hline 5 & Kiterasu & Minawa & Japón & 2017 & 70,00 & OFA & Servicios \\
\hline 6 & Pequeña Casa en el Ferry & Vinalhaven & Estados Unidos & 2014 & 81,00 & Go Logic & Vivienda \\
\hline 7 & Multiply & Londres & Reino Unido & 2018 & 114,92 & Waugh Thistleton & Exhibición \\
\hline 8 & Minimod Curucaca & Curucaca & Brasil & 2018 & 120,00 & MAPA & Vivienda \\
\hline 9 & The Smile & Londres & Reino Unido & 2016 & 136,00 & Alison Brooks & Exhibición \\
\hline 10 & Casa Escobar & San Pedro & Chile & 2013 & 138,00 & Paulina Escobar & Vivienda \\
\hline 11 & Casa Atelier Jones & Seattle & Estados Unidos & 2015 & 139,00 & Atelier Jones & Vivienda \\
\hline 12 & Oficinas Santo & Shiga & Japón & 2017 & 139,00 & Junichi Cato \& Assoc. & Servicios \\
\hline 13 & Casa Rave & Oudenaarde & Belgica & 2014 & 172,00 & Tom Maihue & Vivienda \\
\hline 14 & Dos torres & Portschach am Worthersee & Austria & 2016 & 172,00 & \begin{tabular}{|l|} 
Sonja Hohengasser \& \\
Juergen Wirnsberger \\
\end{tabular} & Vivienda \\
\hline 15 & Casa Sant Julia & Sant Cugat & España & 2014 & 191,00 & $\begin{array}{l}\text { Alejandro Sainz, Marianne } \\
\text { Verdoux } \\
\end{array}$ & Vivienda \\
\hline 16 & Casa Cats & San Bernardo & Chile & 2015 & 180,00 & $\begin{array}{l}\text { Cecilia Poblete, Valentina } \\
\text { Torres, Ignacio Rojas } \\
\end{array}$ & Vivienda \\
\hline 17 & Skilpod \#150 & Ham & Belgica & 2016 & 280,00 & Massimo Pignanelli & Vivienda \\
\hline 18 & Centro Deportes Nauticos & Formentera & España & 2019 & 345,00 & Maria Castelo Martinez & Servicios \\
\hline
\end{tabular}

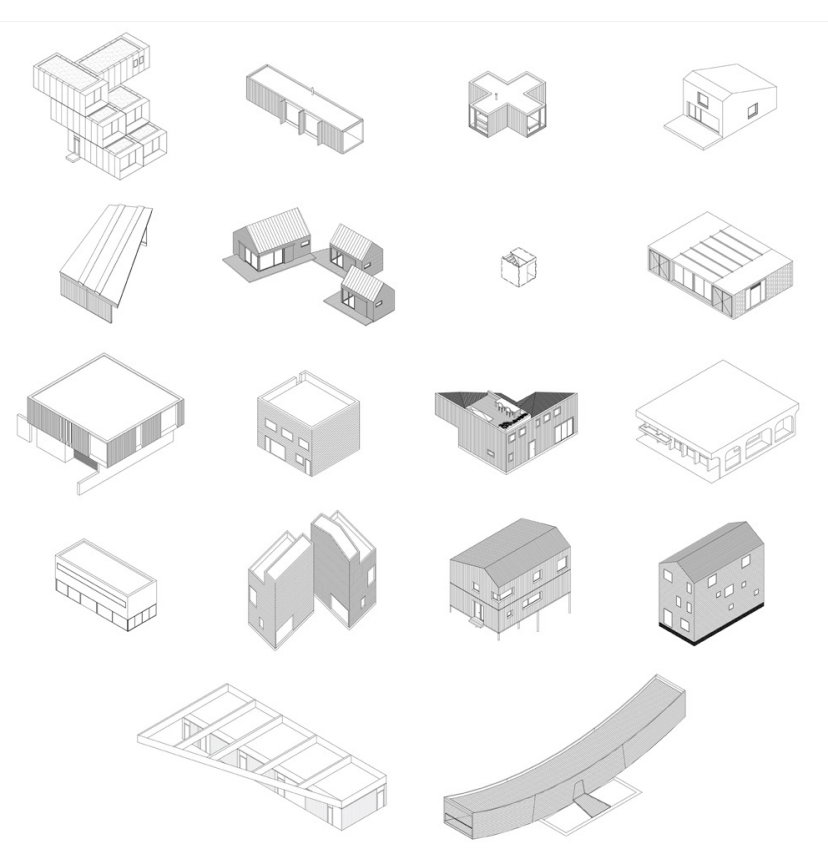

Fig.2. Axonometrías de los Edificios Analizados (elaboración propia).

Con los antecedentes recolectados, se revisaron y midieron condiciones específicas de los distintos edificios. En particular de tres ámbitos; modulación, fenestraciones y dimensiones de las placas; con el fin de disponer informaciones generales del diseño y proceso constructivo. En el primer ámbito sobre modulación se revisaron primero las medidas totales del volumen habitable, basado en las distancias transversales entre ejes estructurales en sus tres dimensiones (Fig.3).
Luego se identificó el módulo típico, considerado como la distancia interior entre ejes estructurales que conformarán espacios principales repetidos, también en las tres dimensiones. Posteriormente se reconoció la cantidad de módulos aplicados en los distintos sentidos, incluyendo en algunos casos espacios laterales a los módulos, en los cuales no se presenta una correspondencia exacta entre los módulos típicos, su cantidad y dimensión total. En la altura, la cantidad de módulos equivalen a los pisos del edificio.

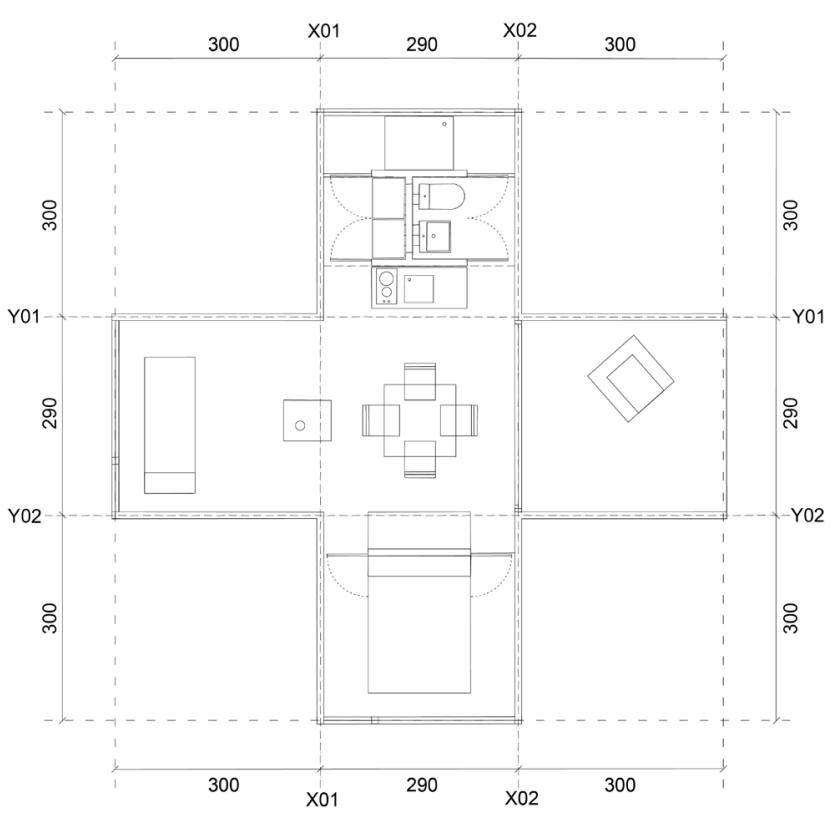

Fig.3. Ejemplo de Análisis de Plantas (elaboración propia).

En el segundo ámbito, se revisó el dimensionamiento de vanos 
en el total de las fachadas (Fig.4), debido a la percepción de una expresión arquitectónica particular. Para lo cual se midieron las superficies completas de las caras expuestas al exterior (entre ejes estructurales), y las medidas interiores entre vanos, determinando el total compuesto entre las distintas fachadas, asi como también los vanos de menor y mayor tamaño (en sus dos medidas). Considerando además algunos casos que el vano se constituye excluyendo la colocación de una placa, incluso en un costado completo en algunas construcciones más pequeñas. Lo que permite mayores dimensiones, pero cautelando que los restantes tramos sean portantes opacos. Como también vanos perforados dentro de placas.
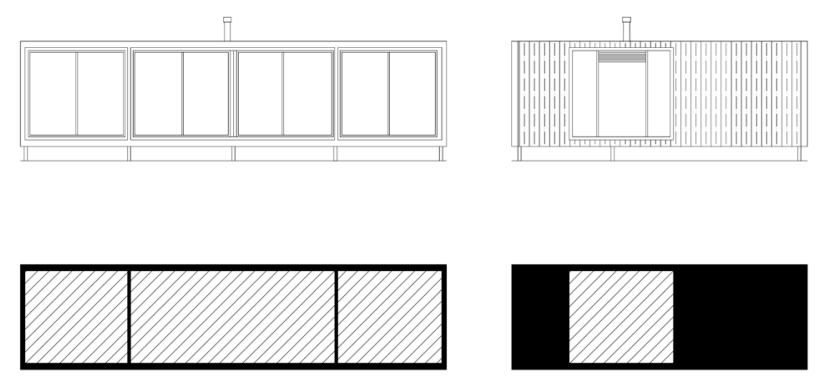

Fig. 4. Ejemplo de Análisis de Fachadas (elaboración propia).

Posteriormente en el tercer ámbito, se revisaron las medidas propias de las placas utilizadas, comenzando por la cantidad de capas y sus espesores, lo que condiciona sus capacidades estructurales y proviene de diferentes convenciones productivas por país. Además de las dimensiones superficiales de las placas, aunque pueden ser variables en algunos casos o en varios fueron medidos desde alzados o fotografías al carecer de documentos proyectuales. Sin embargo, este antecedente es relevante porque incide en la modulación, como también expresa el proceso productivo en cuanto a transporte y montaje.

También se recolectaron antecedentes de los sistemas de montaje y conexión, así como de revestimientos y aislación, que son aspectos cruciales para la ejecución y terminación arquitectónica, pero presentan una variedad de soluciones. Así como, de estrategias compositivas y condiciones espaciales de los edificios estudiados, que son analizados cualitativamente.

Los datos métricos recolectados fueron revisados posteriormente mediante algunas cuantificaciones básicas, para determinar medias y dispersiones, intentando reconocer tendencias principales, como también casos especiales. Además de correlaciones básicas, especialmente entre condiciones de las placas y modulaciones para establecer capacidades específicas. Culminando con una caracterización general del trabajo de investigación.

\section{CONDICIONES CONSTRUCTIVAS DE LOS TA- BLEROS CLT}

Hoy en día el mayor volumen de CLT comercializado a nivel mundial es producido en Europa, siendo Austria, Alemania y Suiza los países que concentran mayor fuerza de manufactura. En Austria específicamente se encuentran las tres principales empresas productoras a nivel mundial: Stora Enso, Binderholz, y KLH. Sin embargo, dado el potencial observado en el CLT como material de construcción, se pronostica que este panorama cambiará vertiginosamente en los próximos años debido a que varios países, entre ellos EE. UU. y Japón se encuentran desarrollando e incrementando sus capacidades de manufactura para posicionarse con fuerza en el mercado mundial (23).

En la producción del CLT se manejan estrictos procesos de manufactura. En Europa estos son regidos por estándares definidos en la norma EN 16351 (24) mientras que en EE.UU y Canadá se maneja el estándar ANSI/APA PRG 320 (25). En cuanto los grados estructurales de estas placas, estos son definidos en Europa por la norma EN 14081-1 (26) (27).

El proceso de manufactura en general se divide en dos fases: en primer lugar, la preparación y tratamiento de la materia prima y en segundo lugar la disposición y encolado del material base (5). En la primera fase, los listones de madera (láminas o lamelas) que conformarán las distintas capas del CLT son usualmente fabricados a partir de madera de baja densidad como el abeto, el alerce, douglas fir o pino (23), luego estos son secados a un $12 \%$ del contenido de humedad y clasificados usando, tanto métodos visuales como métodos mecánicos. El grado de clasificación mecánica mayormente usado es el C24, siendo también usados, en menor porcentaje, los grados $\mathrm{C} 18$ Y C16. En placas de CLT de grandes dimensiones las láminas longitudinales son conformadas por piezas de madera clasificada, ensambladas longitudinalmente mediante uniones dentadas (finger-joint).

En la segunda fase del proceso las lamelas son unidas de canto para conformar las distintas capas que constituirán la placa de CLT. Para esto, en la mayoría de los casos se usan distintos tipos de adhesivos de uso estructural . Existen soluciones donde los listones de las capas interiores son colocados sin adhesión de canto, sin embargo, estas dependen del nivel de hermeticidad requerida en la placa. Una vez conformadas las capas, estas son unidas mediante el uso de adhesivos, con una orientación de lamelas a $90^{\circ}$ con respecto a la otra, lo que le confiere al material alta resistencia y estabilidad dimensional. El proceso de encolado se hace mediante la aplicación

Tabla 2. Medidas Constructivas de los Tableros CLT

\begin{tabular}{|c|c|c|c|c|c|c|}
\hline \multirow{2}{*}{ Empresa } & & Largo & Ancho & Grosor & \multirow{2}{*}{$\mathrm{N}^{\circ}$ de capas } & \multirow{2}{*}{$\begin{array}{c}\text { Ancho de } \\
\text { Lamelas (mm) }\end{array}$} \\
\hline & & (m) & (m) & $(\mathbf{m m})$ & & \\
\hline \multirow{2}{*}{ Stora Enso } & Paredes & 16 max. & 2.95 max. & 60 a 120 & 3-may & 20 y 40 \\
\hline & Losas & 16 max. & o.6o hasta 3.20 & 60 a 320 & $3-5-7-8$ & 20 y 40 \\
\hline \multirow{2}{*}{ BinderHolz } & Paredes & 22 & 3.50 & 60 a 200 & 3-may & $20-30-35-40$ \\
\hline & Losas & 20 & 1.25 & 60 a 340 & $3 / 5 / 07$ & $20-30-35-40$ \\
\hline \multirow{2}{*}{ KLH } & Paredes & 16.5 max. & 2.40 a 2.95 & 57 a 158 & 3 у 5 & $40-34-30-19$ \\
\hline & Losas & 16.5 max. & 2.40 a 2.95 & 60 a 320 & $3-5-7-8$ & $40-34-30-19$ \\
\hline
\end{tabular}


Tabla 3. Resumen de Medidas Registradas.

\begin{tabular}{|c|c|c|c|c|c|c|c|c|c|c|c|c|c|c|c|c|c|c|c|c|c|}
\hline & CASOS & & $\mathbf{1}$ & 2 & 3 & 4 & 5 & 6 & 7 & 8 & 9 & 10 & 11 & 12 & 13 & 14 & 15 & 16 & 17 & 18 & in \\
\hline & $\begin{array}{l}\text { MODULACION } \\
\text { ESPACIAL } \\
\end{array}$ & Unidad & & & & & & & & & & & & & & & & & & & \\
\hline \multirow{3}{*}{ Total } & Largo & $\mathrm{ml}$ & 8,0 & 15 & 9,6 & 8 & 10,7 & 5,24 & 12,5 & 13,4 & 34 & 11,2 & 9,2 & 10 & 14 & 8 & 10,9 & 11,4 & 12 & 17,9 & 12,3 \\
\hline & Ancho & $\mathrm{ml}$ & 4,0 & 3,4 & 3,2 & 5,7 & 7,9 & 3,5 & 11,3 & 9,3 & 4,5 & 11,2 & 7,5 & 11,94 & 5,5 & 6 & 5,4 & 7,68 & 12 & 6,4 & 7,0 \\
\hline & Altura & $\mathrm{ml}$ & 2,9 & 3,2 & 3,2 & 5,6 & 2,8 & 2,4 & 9,1 & 3,2 & 3,5 & 7,5 & 6,45 & 3,1 & 5,5 & 2,5 & 10 & 6,12 & 6,15 & 3 & 4,8 \\
\hline \multirow{3}{*}{ Modulo } & Ancho modulo tipo & $\mathrm{ml}$ & 4,0 & 3,2 & 3,2 & 2,64 & 4 & 3,3 & 2,6 & 3,3 & 4,5 & 3,3 & 3,5 & 3 & 3,8 & 3 & 3,38 & 3,28 & 3,2 & 3,5 & 3,4 \\
\hline & Largo & $\mathrm{ml}$ & 8,0 & 15 & 3,2 & 5,7 & 10 & 3,65 & 2,6 & 8,1 & 12 & 8,5 & 3,6 & & 5,5 & 6 & 2,48 & 8,82 & 10,6 & 6,4 & 6,7 \\
\hline & Alto piso-piso tipo & $\mathrm{ml}$ & 2,4 & 2,8 & 2,8 & 2,7 & 3,6 & 2,4 & 2,6 & 3,1 & 3,5 & 3,2 & 2,4 & 3,1 & 2,3 & 2,5 & 2,66 & 2,6 & 3 & 3 & 2,8 \\
\hline \multirow{4}{*}{ Trazado } & $\begin{array}{l}\text { Cantidad Módulos } \\
\text { Longitud }\end{array}$ & Un. & 1,0 & 1 & 3 & 1 & 1 & 2 & 5 & 1 & 3 & 1,0 & 3 & 4 & 4 & 1 & 3 & 2 & 1 & 10 & 2,6 \\
\hline & $\begin{array}{l}\text { Cantidad Módulos } \\
\text { Ancho }\end{array}$ & Un. & 2,0 & 1 & 3 & 3 & 2 & 1 & 4,5 & 4 & 1 & 2,0 & 2 & 3 & 1 & 3 & 2 & 2 & 3 & 1 & 2,3 \\
\hline & Cantidad de Pisos & Un. & 1,0 & 1 & 1 & 2 & 1 & 1 & 3 & 1 & 1 & 2,0 & 2 & 1 & 2 & 4 & 3 & 2 & 2 & 1 & 1,7 \\
\hline & FENESTRACION & & & & & & & & & & & & & & & & & & & & - \\
\hline \multirow{7}{*}{ Vanos } & Superficie Fachadas & $\mathrm{m}^{2}$ & 96,0 & 116,48 & 100 & 127,5 & 86,2 & 40 & 388 & 145,28 & 234 & 229 & 204,4 & 191.08 & 148,34 & 196 & 268,5 & 241 & 134,4 & 164 & 162,2 \\
\hline & Superficie Vanos & $\mathrm{m}^{2}$ & 13,1 & 33,6 & & 20,8 & 2 & 8,04 & 95,6 & 72,8 & 23 & 21,0 & 25,4 & 61.95 & 12,5 & 15 & 35,9 & 37 & 33,6 & 58.65 & 25,0 \\
\hline & Vano ancho mayor & $\mathrm{ml}$ & 4,0 & 3 & 3,2 & 5 & 0,2 & 2,24 & 1.1 & 6,4 & 4,5 & 3,0 & 2,8 & 3,9 & 13,1 & 4 & 1,3 & 3 & 3,2 & 3,75 & 3,7 \\
\hline & Vano ancho menor & $\mathrm{ml}$ & 0,1 & 3 & 3,2 & 0,75 & 0,1 & 1 & 0,77 & 3,2 & 4,5 & 0,6 & 0,65 & 0,73 & 0,95 & 1 & 0,65 & 0,8 & 0,6 & 1 & 1,3 \\
\hline & Vano alto mayor & $\mathrm{ml}$ & 2,4 & 2,8 & 2,8 & 2,2 & 0,2 & 1,9 & 2.2 & 2,8 & 2,7 & 2,2 & 2 & 2,6 & & 2,2 & 2 & 2,65 & 2,4 & 3 & 2,0 \\
\hline & Vano alto menor & $\mathrm{ml}$ & 0,7 & 2,8 & 2,8 & 0,8 & 0,1 & 50 & 0,8 & 2,8 & 2,7 & 0,3 & 0,8 & 0,95 & & 1,2 & 0,65 & 0,8 & 2,4 & 3 & 4,1 \\
\hline & CONSTRUCCION & & & & & & & & & & & & & & & & & & & & - \\
\hline \multirow{3}{*}{ Muro } & Cantidad de capas & Un. & $\mathrm{s} / \mathrm{a}$ & 3 & 3 & 5 & 7 & 3 & 3 & $\mathrm{~s} / \mathrm{a}$ & $5 / 7$ & 3,0 & 5 & 5 & 5 & 3 & 3 & 3 & 5 & 3 & 3,3 \\
\hline & Espesor de capa & $\mathrm{mm}$. & $\mathrm{s} / \mathrm{a}$ & $\mathrm{s} / \mathrm{a}$ & 3,5 & s/a & s/a & 3,5 & s/a & s/a & 2,5 & s/a & $\mathrm{s} / \mathrm{a}$ & 4 & s/a & s/a & s/a & 3,5 & $\mathrm{~s} / \mathrm{a}$ & 3.5 & 0,9 \\
\hline & Espesor de placa & $\mathrm{mm}$ & s/a & $30-60$ & 11,5 & 150 & 30 & 105 & 99 & $\mathrm{~s} / \mathrm{a}$ & $12,5 / 17,5$ & 30,0 & 150 & 200 & 158 & 30 & 10 & 105 & 30 & 10,5 & 62,2 \\
\hline \multirow{3}{*}{ Tabiques } & Cantidad de capas & Un. & $\mathrm{s} / \mathrm{a}$ & $\mathrm{s} / \mathrm{a}$ & 3 & s/a & 3 & 3 & $\mathrm{~s} / \mathrm{a}$ & $\mathrm{s} / \mathrm{a}$ & s/a & s/a & 3 & 3 & 3 & 3 & $\mathrm{~s} / \mathrm{a}$ & 3 & 5 & 3 & 1,8 \\
\hline & Espesor de capa & $\mathrm{mm}$. & s/a & $\mathrm{s} / \mathrm{a}$ & 3,5 & s/a & s/a & 3,5 & s/a & s/a & s/a & $\mathrm{s} / \mathrm{a}$ & s/a & 4 & s/a & s/a & s/a & 3,5 & s/a & 3.5 & 0,8 \\
\hline & Espesor de placa & $\mathrm{mm}$. & $\mathrm{s} / \mathrm{a}$ & s/a & 11,5 & 10 & 30 & 105 & s/a & s/a & s/a & $\mathrm{s} / \mathrm{a}$ & 90 & 12 & 94 & 30 & s/a & 105 & 30 & 10,5 & 29,3 \\
\hline \multirow{2}{*}{$\begin{array}{l}\text { Placas } \\
\text { Muro }\end{array}$} & Ancho & $\mathrm{mt}$ & $\mathrm{s} / \mathrm{a}$ & 3 & 2,8 & $2-2,9$ & 3 & 2,4 & 2,6 & s/a & 3,5 & 1,7 & 1,2 & 3.1 & 2,5 & 3 & 1,3 & 2,6 & 3,2 & 2,6 & 2,0 \\
\hline & Largo & $\mathrm{mt}$ & $\mathrm{s} / \mathrm{a}$ & 15 & 3,2 & 5,85 & 12 & $5,14 / 3,10$ & 2,6 & $\mathrm{~s} / \mathrm{a}$ & $13,6 / 10,1$ & 2,5 & 2,4 & 1,8 & 14 & 6 & 2,45 & 11,4 & 12 & 3 & 5,2 \\
\hline \multirow{2}{*}{$\begin{array}{l}\text { Placas } \\
\text { Losa }\end{array}$} & Ancho & $\mathrm{mt}$ & $\mathrm{s} / \mathrm{a}$ & $\mathrm{s} / \mathrm{a}$ & 2,8 & $\mathrm{~s} / \mathrm{a}$ & $\mathrm{s} / \mathrm{a}$ & 2,4 & s/a & s/a & 4,5 & $\mathrm{~s} / \mathrm{a}$ & s/a & 1,8 & $\mathrm{~s} / \mathrm{a}$ & $\mathrm{s} / \mathrm{a}$ & $\mathrm{s} / \mathrm{a}$ & 3,9 & $\mathrm{~s} / \mathrm{a}$ & 2,6 & 1,0 \\
\hline & Largo & $\mathrm{mt}$ & s/a & s/a & 3,2 & s/a & s/a & 4,85 & s/a & s/a & $13,6 / 10,1$ & $\mathrm{~s} / \mathrm{a}$ & s/a & 5 & s/a & s/a & s/a & 4,08 & s/a & 7 & 1,3 \\
\hline \multirow{3}{*}{ Losa } & Cantidad de capas & Un. & s/a & 3 & $\mathrm{~s} / \mathrm{a}$ & 5 & 11 & $\mathrm{~s} / \mathrm{a}$ & 3 & $\mathrm{~s} / \mathrm{a}$ & 7 & 4,0 & 5 & 3 & 5 & 5 у 7 & 5 & 5 & 5 & 5 & 3,7 \\
\hline & Espesor de capa & $\mathrm{mm}$ & $\mathrm{s} / \mathrm{a}$ & $\mathrm{s} / \mathrm{a}$ & s/a & s/a & $\mathrm{s} / \mathrm{a}$ & s/a & $\mathrm{s} / \mathrm{a}$ & s/a & s/a & $\mathrm{s} / \mathrm{a}$ & $\mathrm{s} / \mathrm{a}$ & 12 & s/a & $\mathrm{s} / \mathrm{a}$ & $\mathrm{s} / \mathrm{a}$ & $\mathrm{s} / \mathrm{a}$ & $\mathrm{s} / \mathrm{a}$ & 3,5 & 0,9 \\
\hline & Espesor de placa & $\mathrm{mm}$ & s/a & $30-50$ & & 150 & 30 & & 99 & $\mathrm{~s} / \mathrm{a}$ & 17,5 & 30,0 & 150 & 4 & 200 & 30 & 16.6 & 17,5 & 30 & 17,5 & 43,1 \\
\hline
\end{tabular}

de presión al frio, siendo los adhesivos más usados el Poliuretano (PUR), la Melanina Urea Formaldehido (MUF) o la Emulsión de Polímero de Isocianato (EPI) (5). Fabricantes como Binderholz afirman que el porcentaje de adhesivo utilizado en sus placas de CLT se encuentra en el orden del $0.6 \%$ con respecto a un $99.4 \%$ de madera (28). Como último paso, luego del proceso de prensado y fraguado del adhesivo, se procede a dimensionar la placa ajustándola a las medidas requeridas. En casos de que las condiciones de uso lo exijan, el CLT es preservado con distintos productos destinados a ello. El proceso más usado es el de la preservación previa de los listones a través de autoclave.

La configuración de la placa de CLT va a depender de la función estructural del mismo (pared o losa). Usualmente en su configuración se dispone un número simétrico de capas con respecto a una capa central, lo que genera una configuración típica de capas impares $(1,26)$. En placas destinadas a paredes, la dirección longitudinal de las lamelas (sentido de las fibras) que conforman las capas exteriores debe seguir el sentido de la carga a compresión a soportar. De igual forma, en las placas dispuestas para losas de entrepiso y techo, las capas exteriores deberán disponerse con el sentido longitudinal se las lamelas en dirección de los apoyos (30).

Con respecto a las dimensiones de las placas, en los EE.UU. y Canadá responden el estándar de fabricación ANSI/APA PRG 320 (25). Las medidas típicas se encuentran en el orden de los $0.6 \mathrm{~m}, 1.20 \mathrm{~m}, 2.40 \mathrm{~m}$ y $3 \mathrm{~m}$ de ancho y $18 \mathrm{~m}$. de largo máximo. El espesor de las placas va a depender de la capacidad de resistencia mecánica requerida y puede llegar hasta los $350 \mathrm{~mm}$ aprox.. Todas estas medidas con una tolerancia máxima del 2\%. Es importante destacar que, en estos países, estas medidas se encuentran condicionadas por regulaciones de transporte (2).

Normalmente los principales productores muestran catálogos donde se especifican las dimensiones estándar de las placas (largo, ancho y espesor), así como sus distintas configuraciones de capas exteriores en función de su uso como pared o losa. Estas son referenciadas usualmente con sus capacidades de resistencia a compresión, carga lateral y módulo de elasticidad, de manera que la selección de estos se haga de acuerdo a un diseño estructural y modular previo. En Europa, los tres principales productores de CLT, Stora Enzo, Binderholz, y KLH, especifican en sus catálogos distintas dimensiones, las cuales se resumen en la tabla 2.

\section{MODULACIONES DE LOS CASOS ESTUDIADOS}

Como se indica previamente, la búsqueda de información de edificios menores ejecutados en CLT por internet permitió identificar 18 casos, descartando una cantidad similar encontrada de proyectos sin información completa y/o con sistemas combinados. Los edificios registrados corresponden mayoritariamente a viviendas unifamiliares, y en menor 
cantidad a servicios o pabellones de exhibición. Realizados en distintas partes de Europa o América, y en un par de casos en Japón, en su totalidad en los últimos cinco años. Esta selección expresa una creciente aplicación de esta tecnología constructiva en el sector residencial individual, que también es utilizado como ejemplo de aplicación del sistema, debido a su magnitud más reducida que las grandes edificaciones y su relativa mayor facilidad de ejecución y exhibición.

Los casos elegidos (a partir de no superar $400 \mathrm{~m} 2$ ), presentan una media de 136,32 m2 en superficie construida, lo que es bastante próximo a la magnitud de una vivienda unifamiliar, aunque con una amplia dispersión (desde 32 a 345 m2), considerando algunas construcciones compactas, y otras de servicios más amplios. También con una amplia variedad en cuanto a área exterior o interior, ya que en algunos corresponde exclusivamente al espacio cerrados, y en otros se adicionan espacios abiertos o son completamente exteriores (como The Smile o Multiply que son pabellones de exhibición). Las dimensiones máximas en planta, presentan una dispersión de 5,24 a $34 \mathrm{mts}$ y de 3,2 a $12 \mathrm{mts}$. En los distintos sentidos, presentan una baja dispersión. Mientras que la altura con una media de 4,8 mts, se extiende mayormente desde 2,5 hasta $10 \mathrm{mts}$, debido a que poseen desde una hasta cuatro plantas. Es decir, son construcciones horizontalmente bastante regulares en una proporción rectangular, y varían mayormente en la vertical.

Según el registro dimensional (Tabla 3), en las construcciones estudiadas se puede reconocer un "modulo tipo" de ancho medio de 3,4 mts. (que varía entre 2,6 a $4 \mathrm{mts}$ ), con un largo medio de 6,7 mts. (que varía desde 2,6 hasta $12 \mathrm{mts}$.). Con una media de 2,6 módulos en la longitud y 2,3 en el ancho, además de 1,7 pisos de altura, con una escasa varianza (aunque mayor diversidad en la altura especifica de los niveles). Lo que permite advertir cierta regularidad en la organización arquitectónica de los ejes principales, en elementos soportantes que configuran recintos. $\mathrm{El}$ ancho entre líneas interiores queda mayormente dispuesto por la medida de luz estructural de un recinto habitable, y se extiende longitudinalmente desde su propia dimensión (conformando una habitación cuadrada mínima), hasta tres a cuatro veces su extensión (Fig.5). Lo que, en algunos casos, conforma la organización total del pequeño edificio (como los Minimod) o se repite una o dos veces en el sentido lateral o frontal.
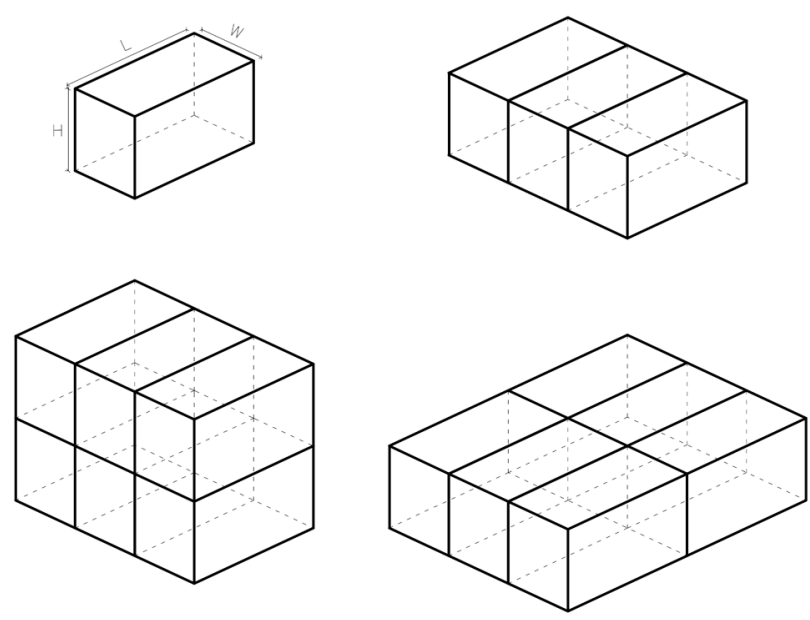

Fig.5. Organización Tridimensional de los Módulos (elaboración propia).
Conformando entonces disposiciones modulares en planta, desde un módulo único, hasta dos o tres por tres o cuatro, es decir hasta una decena de módulos rectangulares; y en altura desde una hasta dos, y en casos hasta tres o cuatro, pero que a la vez utilizan menos en planta. Por tanto, nunca superan la veintena de módulos espaciales. Esta "cuadricula tridimensional” establece la organización espacial y soportante del edificio. Lo cual enfatiza su importancia como sistema constructivo y arquitectónico. Es relevante considerar así mismo, la diversidad de ésta estructura modular, ya que en edificios de destinos, materialidad y magnitudes similares no se adscribe a medidas únicas, ni muy cercanas, ni tampoco a configuraciones equivalentes. Esta selección de casos de épocas, requerimientos y construcción similar, presentan una organización implícita con ciertas propiedades comunes, pero una configuración diferente. Planteando de este modo, un balance entre la regularidad constructiva, necesaria para la racionalidad de la ejecución, y la diversidad para adaptarse a condiciones particulares y otorgar una variedad expresiva.

A su vez, las dimensiones y organización geométrica de los casos estudiados revelan magnitudes que corresponden a requerimientos ocupables convencionales y capacidades regulares del sistema constructivo con tableros CLT. Expresando por tanto una sincronía entre posibilidades relevantes de aplicación y de producción. Como también la muestra analizada presenta una variedad de configuración exterior y disposición interior, que permite reconocer una flexibilidad arquitectónica, con resultados singulares y atractivos.

Respecto a las fachadas y aperturas, los edificios estudiados presentan una superficie media de paramentos exteriores de $162,2 \mathrm{~m} 2$, con una relación de 1,25 sobre la media de superficie construida, que corresponde a un valor normal para pequeñas construcciones; con una superficie media de vanos de $25 \mathrm{~m} 2$, que corresponde a una proporción de 15,39\%, que es una relación reducida para viviendas o pabellones de exposición. Esta proporción estrecha, que se evidencia en la configuración mayormente opaca de los volúmenes arquitectónicos, corresponde probablemente a requerimientos de resistencia estructural, costo constructivo o aislación energética (ya que los casos se sitúan principalmente en climas templados).

Las dimensiones de los vanos presentan, sin embargo, una notable variedad, con medias distantes entre los mayores y menores anchos, y en la diversidad de éstas medidas, aunque no tanto en los altos. En varios de los edificios se reconoce en todo caso, una regularidad entre sus vanos, manteniendo magnitudes o formas similares, o notablemente distintos entre vanos mayores y menores, o concentrados en algunos de los planos de fachadas. Utilizando precisamente esta condición, para plantear una singular expresión arquitectónica en el exterior, como también en el interior respecto a la extensión visual de los espacios. De modo, nuevamente de compensar restricciones constructivas, con una disposición arquitectónica.

En las dimensiones específicas de los tableros fue más difícil recabar información, correspondiendo la mayor parte a interpretaciones de fotografías y en unos pocos casos a antecedentes numéricos de la documentación o respuestas de proyectistas que validaron la información recopilada. En todo caso, se advierte una relevante regularidad en los espesores de capas (mayormente de 30 o $50 \mathrm{~mm}$.) y la cantidad de capas (3 o 5, y algunas de 7 u 11 en losas). Lo que evidencia la utilización de 
las magnitudes medias de las variedades productivas (Fig. 6). Las medidas generales de los tableros presentan una mayor dispersión, con anchos desde 1,2 hasta $3 \mathrm{mts}$. y largos desde 2,4 hasta $15 \mathrm{mts}$., pero que corresponden principalmente a distintas situaciones de montaje en fábrica (con tableros de mayor tamaño) o en obra (con tableros menores), que usualmente son divisiones de los primeros. Considerando que la producción generalmente se realiza en tableros de dimensiones mayores para optimizar la ejecución.

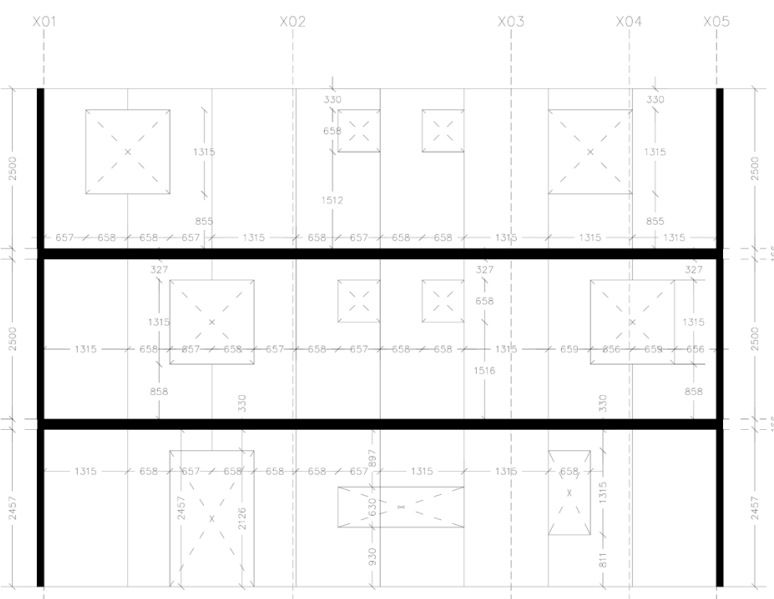

Fig.6. Ejemplo de Despiece de Tableros (elaboración propia).

\section{APLICACION DE LOS TABLEROS CLT}

Para comprender cabalmente la utilización de los tableros CLT en las edificaciones analizadas, es relevante también revisar otras consideraciones constructivas. En particular sobre la configuración estructural, el complemento de atributos de habitabilidad y disposiciones arquitectónicas.

Las construcciones revisadas configuran estructuras espaciales de pisos continuos y muros cortantes, con un comportamiento tridimensional solidario entre planos horizontales y verticales (mayormente ortogonales entre sí). Lo que requiere afianzar los tableros del mismo plano y perpendiculares, así como la base. Para esto utilizan en un par de casos, ensambles entre paneles, y en la mayoría, herrajes metálicos y/o tornillos de profundidad. Esta estrategia es el procedimiento constructivo más regular en la edificación con paneles CLT, aunque por la diversidad de los diseños se advierte una variedad de configuraciones. Desde construcciones con un prisma rectangular y elementos interiores menores (como Catucaba 1, The Smile, Dyson o Casa 2020), o varios prismas adjuntos (como Curucaca, Casa Escobar, Santo, Formentera, etc). Con algunos casos singulares, como Multipy con planos desplazados; Kiterasu o Atelier Jones con planos diagonales y Dos Torres o Casa Ferry con prismas dobles separados.

La estrategia de planos tridimensionales resistentes implica preservar gran parte de las superficies, y como se ha mencionado, minimizar los vanos en fachadas, y también reducir aperturas en pisos o losas (huecos de escaleras). Lo que es hábilmente utilizado por los arquitectos de estos proyectos, que concentran aberturas, regularizan medidas o dejan algunos planos vidriados ajenos a la estructura, para expresar una mayor permeabilidad. Con vanos muy reducidos combinados con otros abiertos (como el Kiretasu) o de una similar forma vertical (Como en Skilpod) y en posiciones extremas (como los Minimod), para enfatizar su capacidad de relación al exterior, aunque seguramente sacrificando regularidad de la iluminación interior.

Por otro lado, es relevante observar como la mayoría de las edificaciones revisadas aplican distintas estrategias de tratamiento o revestimiento sobre los paneles de CLT con la finalidad de protegerlos principalmente del fuego, la humedad y rayos ultravioleta. Aun así, se advierte un interés en algunos de los proyectos, especialmente en interiores, de preservar su apariencia natural del CLT. Incluso por el exterior en algunos casos, como The Smile, Multiply y Kiterasu, aparentemente por condiciones temporales de exhibición. Por otro lado, para asegurar la protección e incrementar el aislamiento térmico y acústico en los paramentos exteriores se utilizan elementos adicionales. Pero la mayoría de los casos se localizan en climas templados y se vinculan a un adecuado desempeño ambiental interior con bajo consumo energético, como también a reducir el mantenimiento, por lo que se aplican tanto productos, como soluciones constructivas (de hasta $20 \mathrm{~cm}$ de espesor) que permiten minimizar la decoloración o deterioro, transmitancia térmica, infiltraciones y el paso del ruido. Entre los materiales más usado para estos fines están la lana mineral (en Casa 2020), lana de vidrio, espumas de poliuretano (usados como aislantes acústicos y térmicos), y estucos, pinturas, sidings de PVC, fibrocemento, planchas de aluminio (en Dyson Institute), o entablados de madera (en Casa del Ferry), los cuales son usados como revestimientos exteriores. (Fig.7).

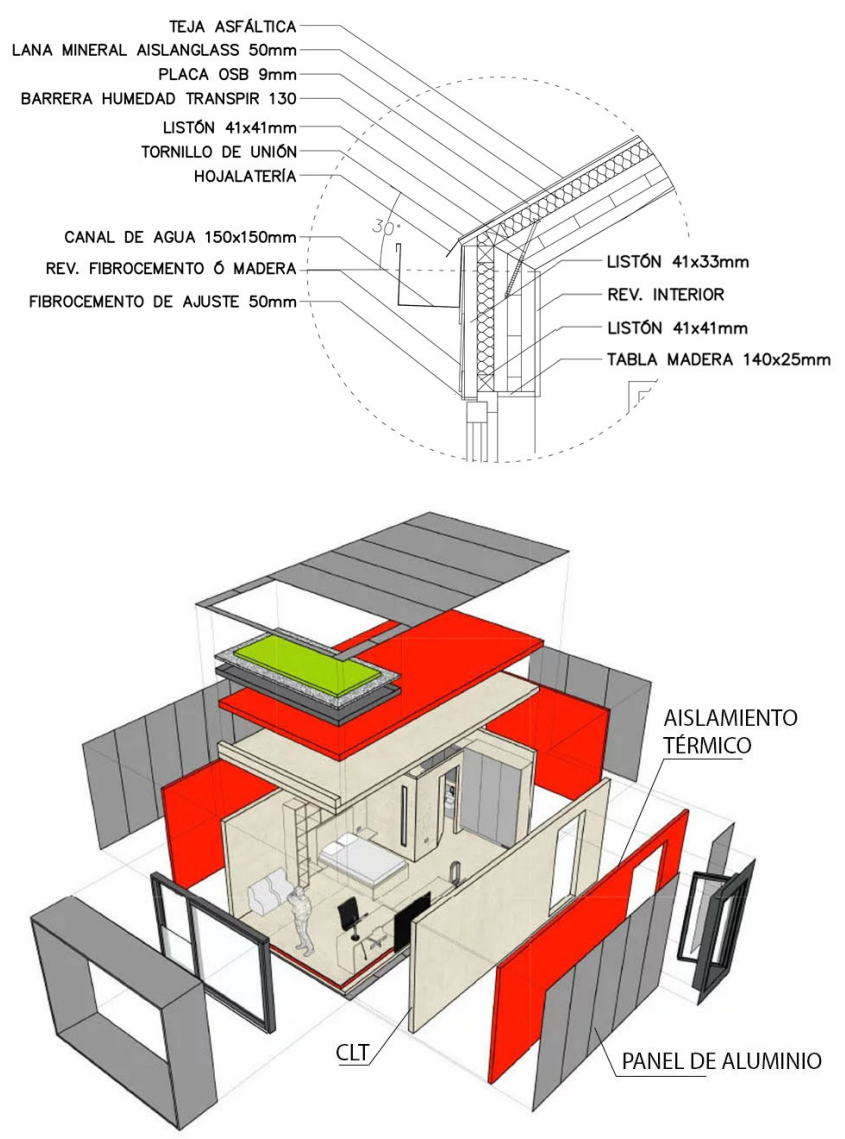

Fig,7. Detalle Constructivo de Casa CATS y Axonometría Explosionada de Dyson Institute (fuentes; elaboración propia e imagen editada de original tomada de: https://www.designboom.com/architecture/wilkinsoneyre-dyson-institute-modular-housing-o6-10-19) 
En relación a la disposición arquitectónica, casi la totalidad de los casos constituyen volúmenes independientes, realzados por su ubicación en entornos desocupados (Fig.8). Ya sea porque son viviendas unifamiliares aisladas en lotes amplios de zonas rurales o suburbios, o pabellones demostrativos que contrastan con su situación urbana. Sólo el Dyson Institute constituye una organización repetitiva. También, configuran y distinguen una forma general de geometría sencilla, usualmente paralelepípedos regulares, en algunos casos, con un costado inclinado (en Kiretasu, Atelier Jones, Formentera), en pares (Ferry y Dos Torres) o repeticiones (Multiply). Esta condición se remarca con cubiertas planas o rehundidas, basamentos separados (incluso transparentes en casa Rave) o tratamientos de vanos o texturas regulares. Con algunas relaciones de costados mayores (longitud en The Smile o Catucaba 1; altura en Escobar, Cats o Dos Torres o San Julia, o ambas horizontales en Santo, Skilpod, Curucaca), que destaca su perfil formal. Utilizando algunos detalles singulares, como la curva general en The Smile, esquinas de vanos en arco en Santo, techumbres inclinadas en Atelier Jones, o voladizos.

En los interiores se advierte a la vez un énfasis casi, al contrario, de extender las visuales y vincular los espacios, minimizando las separaciones y relacionando actividades, especialmente en un mismo nivel horizontal. Colocando vanos de puertas y circulaciones en las esquinas o costados (para mantener la continuidad del muro lateral), y alineando circulaciones o recintos contiguos en la mayor dimensión interior para prolongar la visión. Acompañado por la disposición de ventanas y vistas exteriores. También los tratamientos de muros interiores, con revestimientos homogéneos o texturas lineales, muebles sencillos y contrastantes, algunos integrados en los paramentos, remarcan esta percepción de la espacialidad.

Por otro lado, la implementación en el proyecto arquitectónico de estas características de modulación, disposición ar-

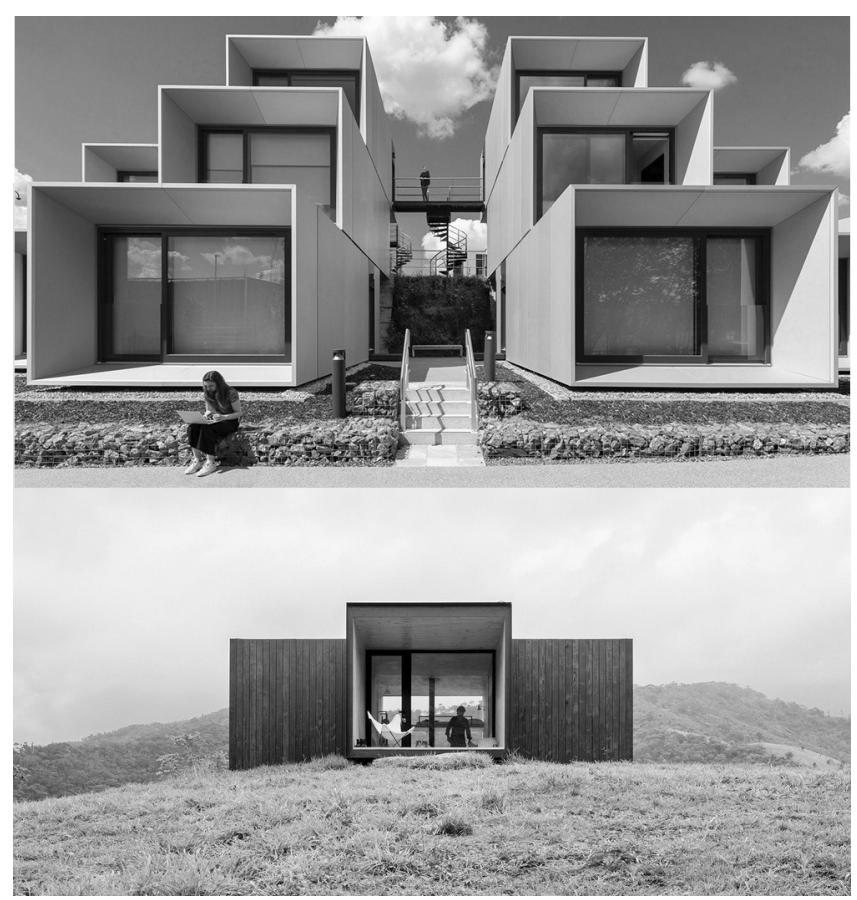

Fig.8. Vistas de Dyson Institute y Casa Catacuba (fuentes; https:// www.dezeen.com/2019/o6/13/dyson-institute-wilkinson-eyre-modular-studaent-housing/ y https://www.archdaily.mx/mx/802166/ minimod-catucaba-mapa?ad_medium=gallery) quitectónica y detallamiento constructivo se pueden realizar en software de modelación de edificios (BIM: Building Information Modeling) mediante dos estrategias concurrentes: a través de definir niveles y ejes como determinaciones de planos de referencia y distanciamientos; y utilizar los mismos elementos constructivos como referencias. Los niveles (horizontales) y los ejes (verticales) funcionan de manera diferente, aunque adecuadas para sugerir modulaciones. Los niveles son planos de situación por el costado inferior de los elementos, y los ejes, trazados de referencia, considerados al eje de los elementos. En ambos casos, permiten vincular dimensiones y acotamientos, pero solamente los niveles son asociados a los elementos. Tampoco se dispone de una estructura general, jerarquías (ejes o niveles principales y secundarios o vinculados) o disposiciones inclinadas, curvas o con alguna figura determinada. Estas propiedades pueden ser programadas (con lenguajes internos o externos), pero es laborioso y su operatividad con el total del modelo es compleja.

Por otro lado, la definición de los tableros como familias BIM (una evolución de los bloques $\mathrm{CAD}$ ) es más versátil, pero también con algunas limitaciones. Se pueden establecer como familias de muros, suelos, losas y/o cubiertas compuestas de tableros con medidas y materiales determinadas; o como familias de formas paramétricas constituidas directamente por los tableros y que permite definir una mayor variedad dimensional, pero sin reconocer su aplicación en el modelo del edificio (no se contabilizan como muros, losas o cubiertas). En ambos casos pueden ser cuantificadas, pero deben ser separados por notas y/o formulas, y considerar piezas especiales. Así mismo los conectores o revestimientos deben ser adicionados o modificados individualmente. Se están desarrollando algunos repertorios o programaciones de tableros CLT para entornos BIM pero aún son limitados y poco verificados. Sin embargo, tiene la posibilidad de integrarse y comunicarse con la maquinaria de elaboración.

\section{DISCUSION}

La documentación de características de los tableros CLT proviene de proveedores del sector, porque se carece todavía de registros estandarizados. Sin embargo, son fabricantes reconocidos con amplia producción ejecutada y sustentado en ensayes o procesos productivos. Por otro lado, los casos estudiados fueron elegidos por un procedimiento aleatorio de búsqueda por internet, al carecer de catastros de obras o una muestra amplia para establecer condiciones. Por lo que ciertamente aparece un sesgo de las capacidades de búsqueda en el periodo temporal y discriminación de la información. Además, la información técnica provista es limitada, lo que se advierte en la carencia de antecedentes precisos del espesor o tipo de placas, o del montaje, que usualmente no se expresa en los medios de divulgación general. De todas maneras, la selección presenta una variedad geográfica y de configuración, que evidencia después una concentración y versatilidad que valida el repertorio estudiado. Es decir, se presentan relevantes atributos comunes y diferencias para constituir una agrupación pertinente de análisis. Aunque ciertamente una selección mayor o condiciones más amplias de superficie o de recursos de búsqueda podría generar una diversidad que amplíe estos hallazgos. Sin embargo, las referencias de otros ejemplos similares y documentaciones de edificios mayores, sugiere que las propiedades detectadas deberían consolidarse.

Por otro lado, se debe reconocer que la recopilación de casos, se basa en medios de divulgación que seleccionan obras ejem- 
plares, y en esta oportunidad que destacan en el uso inicial de una nueva tecnología constructiva. Por tanto, esta muestra reúne capacidades y motivaciones singulares para aplicar los tableros y la producción restante, ya sea actual o futura, aunque considere características similares, puede tener una utilización más regular o menos expresiva. Sin embargo, esta tendencia justifica también la selección como aplicaciones señeras e ilustrativa de las posibilidades arquitectónicas, estimulando el desarrollo constructivo del sistema de paneles CLT.

La aplicación de los paneles en las edificaciones analizadas evidencia un ajustamiento de las plantas y conformación volumétrica a una estructura espacial con modulaciones tridimensionales y predominio superficies opacas. Pero también una progresiva exploración en el diseño de dimensiones, disposición de recintos, relaciones internas y tratamientos que otorguen variabilidad y expansión espacial. Como también en los volúmenes generales y fachadas exteriores, demostrar una expresión contundente y novedosa, con revestimientos y entornos que realcen esta condición. De modo, que la utilización de los paneles CLT en estas obras arquitectónicas constituye una posibilidad de eficiencia constructiva y responsabilidad ambiental, pero también de mejoramiento de calidad interior y proyección exterior.

\section{CONCLUSIONES}

La revisión bibliográfica y estudio de casos de construcciones menores ejecutadas en tableros contralaminados de madera (CLT) releva una interesante convergencia de propiedades técnicas y versatilidad arquitectónica, que pueden impulsar la industria del sector. Además, según el estudio realizado se puede sugerir a la industria de paneles CLT disponer, especialmente para las edificaciones menores, de placas con las magnitudes mencionadas previamente, es decir en un rango central de producción, para promover la ejecución con éste sistema constructivo. Como también para instaladores disponer de conectores o procesos de ensamble, transporte y montaje relacionado con este repertorio de paneles, y ordenación en estructuras tridimensionales; considerando diferentes alternativas de revestimiento y conformación arquitectónica.

El análisis de los casos, en cuanto a sus características arquitectónicas y dimensionales, evidencia una concentración en edificaciones residenciales de un piso (a lo más dos o tres), con un o dos módulos de extensión, y entrepisos regulares, utilizando placas de espesores medios ( 3 a 5 capas de 30 $\mathrm{mm}$.) y longitudes medianas ( $1 \mathrm{a} 3 \mathrm{mts}$ de ancho, y 6 a $12 \mathrm{mts}$. de extensión), con un alto volumen de material respecto a la superficie construida $(0,2 \mathrm{~m} 3 / \mathrm{m} 2)$. Lo que evidencia un potencial de mercado focalizado, que puede guíar la producción y abaratar los costos por la masificación y competitividad. Promoviendo que los fabricantes preparen y ofrezcan particularmente estas tipologías de placas y aplicaciones.

También el estudio de los casos muestra una variedad de configuraciones arquitectónicas, en el uso de los módulos y dimensiones básicas, como en la disposición general y ordenación particular de recintos, alejándose de diseños repetitivos o simples. Utilizando remates o costados diagonales, dobles alturas, relaciones transversales y extensiones longitudinales de los recintos y paramentos, para generar efectos espaciales novedosos, que realzan la flexibilidad constructiva de las placas. Utilizando hábilmente la disposición de vanos, con aberturas amplias en algunos frentes, y otras pequeñas o contrastadas con planos opacos, para lidiar con la magnitud de cerramiento estructural que requieren los tableros. Así mismo, disponiendo revestimientos exteriores, tratamientos interiores, elementos menores, empalmes de tableros o de recintos que destacan la expresividad del material y de los espacios configurados. Constituyéndose en ejemplos inspiradores para aplicar arquitectónicamente esta nueva tecnología con grandes ventajas constructivas y ambientales.

\section{AGRADECIMIENTOS}

Se agradece la colaboración en este trabajo de los siguientes estudiantes de la carrera de Arquitectura de la Universidad del Bío-Bío (Concepción, Chile), Camila Aburto, Tomas Acuña, Javiera Aravena, Francisca Avilés, Giselle Carrasco, Dayanna Gaete, Kevin Ibañez, Elias Inzunza, Maicol Mansilla, Sofia Montecinos, Scarlet Mora, Denisse Navarrete, Damary Parra, Francisco Roa, Constanza Rojas y Francisca Romero que recopilaron y redibujaron planos de algunos casos analizados, y Francisco Galindo en la coordinación. También al Grupo de Investigación en Gestión y Diseño Integrado de Edificios de la Universidad del Bío-Bío.

\section{REFERENCIAS / REFERENCES}

(1) Albee, R.R., Muszyński, L., Hansen, E.N., Knowles, C.D., Larasatie, P. y Guerrero, J.E.(2018). Recent developments in global cross-laminated timber (CLT) market. WCTE 2018 - World Conference on Timber Engineering, no. August.

(2) Jelec, M., Varevac, D. y Rajcic, V. (2018). Cross-laminated timber (CLT) - A state of the art report. Journal of the Croatian Association of Civil Engineers, vol. 70, no. 02, pp. 75-95. Issn 03502465. Doi 10.14256/jce.2071.2017.

(3) Viotto U. (2013) El tablero contralaminado. (Tesina del Máster oficial universitario "Tecnología en la arquitectura") Barcelona: Universidad Politécnica de Cataluña.

(4) González, P.; Saavedra, E., Pérez, E. Burgos, C. Piña, F. y Wagner, M. (2014) Sistema constructivo en madera contralaminada para edificios. Vol. 1. Santiago de Chile: Universidad de Santiago de Chile.

(5) Jeleč M, Varevac D, Rajčić V. (2018) Cross-laminated timber (CLT) - a state of the art report, GRAĐEVINAR 70 (2), 75-95

(6) Vidal, R.; Sánchez-Pantoja, N.; Martínez, G. (2019). Life cycle assessment of a residential building with cross-laminated timber structure in Granada-Spain. Informes de la Construcción, 71(554)

(7) Orlowski K., Automated manufacturing for timber-based panelised wall systems, Automation in Construction Vol.109, 102988

(8) Roldán, F. (2012) Método de modulación y dimensionado de la arquitectura histórica, Congreso Internacional de Innovación en el Patrimonio, Valladolid: Junta de Castillo y León

(9) Durand J.N.L. (1813) Nouveau précis des leçons d'architecture : données a l'Ecole impériale polytechnique,Paris:Fantin.

(10) Martínez Corbella, C. (1992) La concepción arquitectónica y la industrialización: teoría general, Santiago de Chile : Universidad de Valparaíso. 
(11) Mac Donell (1999) Manual de construcción industrializada Disponible en: https://cetarq.com/wp-content/ uploads/2016/05/industrializada.pdf.

(12) Le Corbusier (1979), Modulor, Madrid: Ed. Poseidon.

(13) Ropero D. y Comas A. (2013) Construcción modular de viviendas y arquitectura, Valencia: Dream, pp. 77.

(14) Richard, R. ( 2006) Industrialised, flexible and demountable building systems: Quality, economy and sustainability. Proceedings of CRIOCM 2006 International Research Symposium on Advancement of Construction Management and Real Estate, Vols 1 and 2, (pp. 1-10), Beijing: Chinese Research Institute of Construction Management.

(15) Eastman, C. (2011) BIM handbook: a guide to building information modeling for owners, managers, designers, engineers, and contractors, New Jersey : John Wiley.

(16) Jensen P. (2015) Product configuration in construction. International Journal of Mass Customisation, vol. 5, no. 1, pp. 73.

(17) Habraken N J (2000) El Diseño de Soportes, Barcelona : Editorial Gustavo Gili.

(18) Bermek M S , Shelden D , T. Gentry R, (2019), A Holistic Approach to Feature-based Structural Mapping in Cross Laminated Timber Buildings, Ecaade-Sigradi Conference, Porto: University of Porto

(19) Viklund, E. (2017) Design approaches in industrialized house building: A creativity perspective. (Tesis Department of Civil, Environmental and Natural Resources Engineering, Industrialized and sustainable construction), Luleå University of Technology.

(20) Da Rocha, C. G.; Formoso C.T. y Tzortzopoulos P. (2015) Adopting product modularity in house building to support mass customisation. Sustainability (Switzerland), vol. 7, no. 5, pp. 4919-4937.

(21) Wikberg, F.; Olofsson, T. y Ekholm, A. (2014) Design configuration with architectural objects: Linking customer requirements with system capabilities in industrialized housebuilding platforms. Construction Management and Economics vol. 32, no. 1-2, pp. 196-207

(22) Echeverria-Quintana, M., (2015). Metodologia del diseño conceptual modular para la selección de variables modulares (Tesis de Doctorado), Barcelona: Universidad Politécnica de Catalunya.

(23) CBI Ministry of Foreign Affairs (2015). Exporting cross laminated timber (CLT) to Western Europe. [en línea], no. Lvl. Disponible en: https://www.cbi.eu/market-information/timber-products/cross-laminated-timber/europe/.

(24) European Committee for Standardization (CEN): Timber structures - Cross laminated timber- Requirements. EN 16351, Brussels, 2015.

(25) APA - The Engineered Wood Association: Standard for Performance Rated Cross-Laminated Timber PRG 320, 2011.

(26) EN 14081-1 Timber structures - Strength gradaed structural timber with rectangular cross section - Part 1: General Requirements, European Committee for Standardization (CEN), Brussels, Belgium, 2005.

(27) Young, T.M., Barbu, M.C., Hindman, D.P., Weissensteiner, J. y Tudor, E.M., (2016). Comparisons of the production standards for Cross laminated timber (CLT) in Europe versus USA. WCTE 2016 - World Conference on Timber Engineering, Viena: Vienna University of Technology.

(28) Binderholz (2019) Solid timber construction manual 2.o, [en línea] Disponible en https://www.massivholzhandbuch. com/en/

(29) Gijzen, R.P.T. (2017) Modular cross-laminated timber buildings. [en línea], Disponible en:https://repository.tudelft. nl/islandora/object/uuid\%3Af687e2cc-86e1-442e-862a2ceb382f7157.

(30) Crampton , A.N.C (2016). Cross Laminated Timb Er : the Future of Mid - Rise Construction, Center for Real Estate Quarterly Report, vol. 10, no. 3, pp. 22-26. 